\title{
Dual Spaces and Hahn-Banach Theorem ${ }^{1}$
}

\author{
Keiko Narita \\ Hirosaki-city \\ Aomori, Japan
}

\author{
Noboru Endou \\ Gifu National College of Technology \\ Gifu, Japan \\ Yasunari Shidama \\ Shinshu University \\ Nagano, Japan
}

\begin{abstract}
Summary. In this article, we deal with dual spaces and the Hahn-Banach Theorem. At the first, we defined dual spaces of real linear spaces and proved related basic properties. Next, we defined dual spaces of real normed spaces. We formed the definitions based on dual spaces of real linear spaces. In addition, we proved properties of the norm about elements of dual spaces. For the proof we referred to descriptions in the article 21. Finally, applying theorems of the second section, we proved the Hahn-Banach extension theorem in real normed spaces. We have used extensively used [17].
\end{abstract}

MSC: 46A22 46E15 03B35

Keywords: dual space; Hahn-Banach extension

MML identifier: DUALSP01, version: 8.1.03 5.23.1204

The notation and terminology used in this paper have been introduced in the following articles: [5], [16], 23], [18, 6], 7], [17, [15], 21], 24], [1], 22, [20], [3], [8], 44, [28], 25], [26], [10], [22], [12], [13], 27], [14], and [9].

\section{Dual Spaces of Real Linear Spaces}

From now on $V$ denotes a non empty real linear space.

Let $X$ be a real linear space. The functor MultFReal* $X$ yielding a function from (the carrier of $\left.\mathbb{R}_{\mathrm{F}}\right) \times($ the carrier of $X$ ) into the carrier of $X$ is defined by the term

(Def. 1) The external multiplication of $X$.

\footnotetext{
${ }^{1}$ This work was supported by JSPS KAKENHI 22300285, 23500029.
} 
Now we state the proposition:

(1) Let us consider a real linear space $X$. Then $\langle$ the carrier of $X$, the addition of $X$, the zero of $X$, MultFReal $* X\rangle$ is a vector space over $\mathbb{R}_{\mathrm{F}}$.

Let $X$ be a real linear space. The functor RLSp2RVSp $X$ yielding a vector space over $\mathbb{R}_{F}$ is defined by the term

(Def. 2) 〈the carrier of $X$, the addition of $X$, the zero of $X$, MultFReal $* X\rangle$.

Let $X$ be a vector space structure over $\mathbb{R}_{\mathrm{F}}$. The functor MultReal $* X$ yielding a function from $\mathbb{R} \times$ (the carrier of $X$ ) into the carrier of $X$ is defined by the term

(Def. 3) The left multiplication of $X$.

Now we state the proposition:

(2) Let us consider a vector space $X$ over $\mathbb{R}_{\mathrm{F}}$. Then $\langle$ the carrier of $X$, the zero of $X$, the addition of $X$, MultReal $* X\rangle$ is a real linear space.

Let $X$ be a vector space over $\mathbb{R}_{\mathrm{F}}$. The functor RVSp2RLSp $X$ yielding a real linear space is defined by the term

(Def. 4) 〈the carrier of $X$, the zero of $X$, the addition of $X$, MultReal $* X\rangle$.

Now we state the propositions:

(3) Let us consider a real linear space $X$, elements $v, w$ of $X$, and elements $v_{1}, w_{1}$ of RLSp2RVSp $X$. If $v=v_{1}$ and $w=w_{1}$, then $v+w=v_{1}+w_{1}$ and $v-w=v_{1}-w_{1}$.

(4) Let us consider a vector space $X$ over $\mathbb{R}_{\mathrm{F}}$, elements $v, w$ of $X$, and elements $v_{1}, w_{1}$ of RVSp2RLSp $X$. If $v=v_{1}$ and $w=w_{1}$, then $v+w=$ $v_{1}+w_{1}$ and $v-w=v_{1}-w_{1}$.

Let $V$ be a non empty real linear space. The functor $\bar{V}$ yielding a strict non empty real linear space is defined by

(Def. 5) There exists a non empty vector space $X$ over $\mathbb{R}_{\mathrm{F}}$ such that

(i) $X=\operatorname{RLSp} 2 \mathrm{RVSp} V$, and

(ii) $i t=\operatorname{RVSp} 2 \operatorname{RLSp} \bar{X}$.

Now we state the proposition:

(5) Let us consider an object $x$. Then $x \in$ the carrier of $\bar{V}$ if and only if $x$ is a linear functional in $V$.

Let $V$ be a non empty real linear space. One can check that $\bar{V}$ is constituted functions.

Let $f$ be an element of $\bar{V}$ and $v$ be a vector of $V$. Note that the functor $f(v)$ yields an element of $\mathbb{R}$. Now we state the propositions:

(6) Let us consider a non empty real linear space $V$ and vectors $f, g, h$ of $\bar{V}$. Then $h=f+g$ if and only if for every vector $x$ of $V, h(x)=f(x)+g(x)$. 
(7) Let us consider a non empty real linear space $V$, vectors $f, h$ of $\bar{V}$, and a real number $a$. Then $h=a \cdot f$ if and only if for every vector $x$ of $V$, $h(x)=a \cdot f(x)$.

(8) Let us consider a non empty real linear space $V$. Then $0 \bar{V}=($ the carrier of $V) \longmapsto 0$.

(9) Let us consider a real linear space $X$. Then (the carrier of $X) \longmapsto 0$ is a linear functional in $X$. Proof: Set $f=($ the carrier of $X) \longmapsto 0$. $f$ is additive by [23, (7)]. $f$ is homogeneous by [23, (7)].

Let $X$ be a real linear space. The linear functionals of $X$ yielding a subset of $\mathbb{R}_{\mathbb{R}}^{(\text {the carrier of } X)}$ is defined by

(Def. 6) Let us consider an object $x$. Then $x \in$ it if and only if $x$ is a linear functional in $X$.

Let $X$ be a real normed space. One can verify that the linear functionals of $X$ is non empty.

Let $X$ be a real linear space. One can verify that the linear functionals of $X$ is non empty and functional.

Let us consider a real linear space $X$. Now we state the propositions:

(10) The linear functionals of $X$ is linearly closed. Proof: Set $W=$ the linear functionals of $X$. For every vectors $v, u$ of $\mathbb{R}_{\mathbb{R}}^{\alpha}$ such that $v, u \in$ the linear functionals of $X$ holds $v+u \in$ the linear functionals of $X$, where $\alpha$ is the carrier of $X$ by [7, (66)], [18, (1)]. For every real number $a$ and for every vector $v$ of $\mathbb{R}_{\mathbb{R}}^{\alpha}$ such that $v \in W$ holds $a \cdot v \in W$, where $\alpha$ is the carrier of $X$ by [7, (66)], [18, (4)].

(11) 〈the linear functionals of $X$, Zero(the linear functionals of $X, \mathbb{R}_{\mathbb{R}}^{\alpha}$ ), Add (the linear functionals of $X, \mathbb{R}_{\mathbb{R}}^{\alpha}$ ), Mult (the linear functionals of $\left.\left.X, \mathbb{R}_{\mathbb{R}}^{\alpha}\right)\right\rangle$ is a subspace of $\mathbb{R}_{\mathbb{R}}^{\alpha}$, where $\alpha$ is the carrier of $X$.

Let $X$ be a real linear space. Note that $\langle$ the linear functionals of $X$, Zero (the linear functionals of $X, \mathbb{R}_{\mathbb{R}}^{\text {(the carrier of } X)}$ ), $\operatorname{Add}($ the linear functionals of $X, \mathbb{R}_{\mathbb{R}}^{(\text {the carrier of } X)}$ ), Mult(the linear functionals of $\left.\left.X, \mathbb{R}_{\mathbb{R}}^{(\text {the carrier of } X)}\right)\right\rangle$ is Abelian add-associative right zeroed right complementable scalar distributive vector distributive scalar associative and scalar unital.

The functor $\bar{X}$ yielding a strict real linear space is defined by the term

(Def. 7) (the linear functionals of $X$, Zero(the linear functionals of $X, \mathbb{R}_{\mathbb{R}}^{\alpha}$ ), Add (the linear functionals of $X, \mathbb{R}_{\mathbb{R}}^{\alpha}$ ), Mult(the linear functionals of $\left.\left.X, \mathbb{R}_{\mathbb{R}}^{\alpha}\right)\right\rangle$, where $\alpha$ is the carrier of $X$.

Observe that $\bar{X}$ is constituted functions.

Let $f$ be an element of $\bar{X}$ and $v$ be a vector of $X$. One can verify that the functor $f(v)$ yields an element of $\mathbb{R}$. Now we state the propositions:

(12) Let us consider a real linear space $X$ and vectors $f, g, h$ of $\bar{X}$. Then $h=f+g$ if and only if for every vector $x$ of $X, h(x)=f(x)+g(x)$. The 
theorem is a consequence of (10).

(13) Let us consider a real linear space $X$, vectors $f, h$ of $\bar{X}$, and a real number $a$. Then $h=a \cdot f$ if and only if for every vector $x$ of $X, h(x)=$ $a \cdot f(x)$. The theorem is a consequence of (10).

(14) Let us consider a real linear space $X$. Then $0_{\bar{X}}=($ the carrier of $X) \longmapsto$ 0 . The theorem is a consequence of (10).

\section{Dual Spaces of Real Normed Spaces}

In the sequel $S$ denotes a sequence of real numbers, $k, n, m, m_{1}$ denote natural numbers, and $g, h, r, x$ denote real numbers.

Let $S$ be a sequence of real numbers and $x$ be a real number. The functor $S-x$ yielding a sequence of real numbers is defined by

(Def. 8) $i t(n)=S(n)-x$.

Now we state the proposition:

(15) If $S$ is convergent, then $S-x$ is convergent and $\lim (S-x)=\lim S-x$.

Let $X$ be a real normed space and $I$ be a functional in $X$. We say that $I$ is Lipschitzian if and only if

(Def. 9) There exists a real number $K$ such that

(i) $0 \leqslant K$, and

(ii) for every vector $x$ of $X,|I(x)| \leqslant K \cdot\|x\|$.

Now we state the proposition:

(16) Let us consider a real normed space $X$ and a functional $f$ in $X$. If for every vector $x$ of $X, f(x)=0$, then $f$ is Lipschitzian.

Let $X$ be a real normed space. One can check that there exists a linear functional in $X$ which is Lipschitzian.

The bounded linear functionals $X$ yielding a subset of $\bar{X}$ is defined by

(Def. 10) Let us consider a set $x$. Then $x \in$ it if and only if $x$ is a Lipschitzian linear functional in $X$.

One can check that the bounded linear functionals $X$ is non empty.

Let us consider a real normed space $X$. Now we state the propositions:

(17) The bounded linear functionals $X$ is linearly closed. Proof: Set $W=$ the bounded linear functionals $X$. For every vectors $v, u$ of $\bar{X}$ such that $v, u \in W$ holds $v+u \in W$ by [5, (56)], (12). For every real number $a$ and for every vector $v$ of $\bar{X}$ such that $v \in W$ holds $a \cdot v \in W$ by [5, (46), (65)], (13).

(18) 〈the bounded linear functionals $X$, Zero(the bounded linear functionals $X, \bar{X})$, Add(the bounded linear functionals $X, \bar{X}$ ), Mult(the bounded linear functionals $X, \bar{X})\rangle$ is a subspace of $\bar{X}$. 
Let $X$ be a real normed space. Let us observe that $\langle$ the bounded linear functionals $X$, Zero(the bounded linear functionals $X, \bar{X}$ ), Add(the bounded linear functionals $X, \bar{X})$, Mult(the bounded linear functionals $X, \bar{X})\rangle$ is Abelian add-associative right zeroed right complementable vector distributive scalar distributive scalar associative and scalar unital.

The $\mathbb{R}$-vector space of bounded linear functionals of $X$ yielding a strict real linear space is defined by the term

(Def. 11) 〈the bounded linear functionals $X$, Zero(the bounded linear functionals $X, \bar{X}), \operatorname{Add}($ the bounded linear functionals $X, \bar{X})$, Mult(the bounded linear functionals $X, \bar{X})\rangle$.

One can check that every element of the $\mathbb{R}$-vector space of bounded linear functionals of $X$ is function-like and relation-like.

Let $f$ be an element of the $\mathbb{R}$-vector space of bounded linear functionals of $X$ and $v$ be a vector of $X$. Note that the functor $f(v)$ yields an element of $\mathbb{R}$. Now we state the propositions:

(19) Let us consider a real normed space $X$ and vectors $f, g, h$ of the $\mathbb{R}$-vector space of bounded linear functionals of $X$. Then $h=f+g$ if and only if for every vector $x$ of $X, h(x)=f(x)+g(x)$. The theorem is a consequence of (17) and (12).

(20) Let us consider a real normed space $X$, vectors $f, h$ of the $\mathbb{R}$-vector space of bounded linear functionals of $X$, and a real number $a$. Then $h=a \cdot f$ if and only if for every vector $x$ of $X, h(x)=a \cdot f(x)$. The theorem is a consequence of (17) and (13).

(21) Let us consider a real normed space $X$. Then $0_{\alpha}=$ (the carrier of $X) \longmapsto 0$, where $\alpha$ is the $\mathbb{R}$-vector space of bounded linear functionals of $X$. The theorem is a consequence of (14) and (17).

Let $X$ be a real normed space and $f$ be an object.

The functor Bound2Lipschitz $(f, X)$ yielding a Lipschitzian linear functional in $X$ is defined by the term

(Def. 12) $\quad f(\in$ the bounded linear functionals $X)$.

Let $u$ be a linear functional in $X$. The functor PreNorms $(u)$ yielding a non empty subset of $\mathbb{R}$ is defined by the term

(Def. 13) $\{|u(t)|$, where $t$ is a vector of $X:\|t\| \leqslant 1\}$.

Let $g$ be a Lipschitzian linear functional in $X$. Observe that $\operatorname{PreNorms}(g)$ is upper bounded.

Now we state the proposition:

(22) Let us consider a real normed space $X$ and a linear functional $g$ in $X$. Then $g$ is Lipschitzian if and only if $\operatorname{PreNorms}(g)$ is upper bounded.

Let $X$ be a real normed space. The bounded linear functionals norm $X$ yielding a function from the bounded linear functionals $X$ into $\mathbb{R}$ is defined by 
(Def. 14) Let us consider an object $x$. Suppose $x \in$ the bounded linear functionals $X$. Then $i t(x)=\sup \operatorname{PreNorms}(\operatorname{Bound} 2 \operatorname{Lipschitz}(x, X))$.

Let us consider a real normed space $X$ and a Lipschitzian linear functional $f$ in $X$. Now we state the propositions:

(23) Bound2Lipschitz $(f, X)=f$.

(24) (The bounded linear functionals norm $X)(f)=\sup \operatorname{PreNorms}(f)$. The theorem is a consequence of (23).

Let $X$ be a real normed space. The functor DualSp $X$ yielding a non empty normed structure is defined by the term

(Def. 15) 〈the bounded linear functionals $X$, Zero(the bounded linear functionals $X, \bar{X})$, Add(the bounded linear functionals $X, \bar{X})$, Mult(the bounded linear functionals $X, \bar{X})$, the bounded linear functionals norm $X\rangle$.

Now we state the propositions:

(25) Let us consider a real normed space $X$. Then (the carrier of $X) \longmapsto 0=$ $0_{\text {Dualsp } X}$. The theorem is a consequence of (21).

(26) Let us consider a real normed space $X$, a point $f$ of DualSp $X$, and a Lipschitzian linear functional $g$ in $X$. Suppose $g=f$. Let us consider a vector $t$ of $X$. Then $|g(t)| \leqslant\|f\| \cdot\|t\|$. The theorem is a consequence of (24).

(27) Let us consider a real normed space $X$ and a point $f$ of DualSp $X$. Then $0 \leqslant\|f\|$. The theorem is a consequence of $(24)$.

(28) Let us consider real normed spaces $X, Y$ and a point $f$ of DualSp $X$. If $f=0_{\text {Dualsp } X}$, then $0=\|f\|$. Proof: $\|f\|=0$ by [23, (45)], [13, (45)], $(25),[23,(7)]$.

Let $X$ be a real normed space. Note that every element of DualSp $X$ is function-like and relation-like.

Let $f$ be an element of DualSp $X$ and $v$ be a vector of $X$. Let us note that the functor $f(v)$ yields an element of $\mathbb{R}$. Now we state the propositions:

(29) Let us consider a real normed space $X$ and points $f, g, h$ of DualSp $X$. Then $h=f+g$ if and only if for every vector $x$ of $X, h(x)=f(x)+g(x)$. The theorem is a consequence of (19).

(30) Let us consider a real normed space $X$, points $f, h$ of DualSp $X$, and a real number $a$. Then $h=a \cdot f$ if and only if for every vector $x$ of $X$, $h(x)=a \cdot f(x)$. The theorem is a consequence of $(20)$.

(31) Let us consider a real normed space $X$, points $f, g$ of DualSp $X$, and a real number $a$. Then

(i) $\|f\|=0$ iff $f=0_{\text {DualSp } X}$, and

(ii) $\|a \cdot f\|=|a| \cdot\|f\|$, and

(iii) $\|f+g\| \leqslant\|f\|+\|g\|$. 
Proof: $\|f+g\| \leqslant\|f\|+\|g\|$ by [13, (45)], (27), [5, (56)], (26). $\|a \cdot f\|=$ $|a| \cdot\|f\|$ by (27), (26), [5, (65), (46)].

Let $X$ be a real normed space. Note that DualSp $X$ is reflexive discernible and real normed space-like.

Now we state the proposition:

(32) Let us consider a real normed space $X$. Then DualSp $X$ is a real normed space.

Let $X$ be a real normed space. Let us note that DualSp $X$ is reflexive discernible real normed space-like vector distributive scalar distributive scalar associative scalar unital Abelian add-associative right zeroed and right complementable.

Now we state the proposition:

(33) Let us consider a real normed space $X$ and points $f, g, h$ of DualSp $X$. Then $h=f-g$ if and only if for every vector $x$ of $X, h(x)=f(x)-g(x)$. The theorem is a consequence of (29).

Let $X$ be a real normed space, $s$ be a sequence of DualSp $X$, and $n$ be a natural number. Let us note that the functor $s(n)$ yields an element of DualSp $X$. Now we state the propositions:

(34) Let us consider a real normed space $X$ and a sequence $s_{1}$ of DualSp $X$. If $s_{1}$ is Cauchy sequence by norm, then $s_{1}$ is convergent. Proof: Define $\mathcal{P}$ [set, set] $\equiv$ there exists a sequence $x_{1}$ of $\mathbb{R}$ such that for every natural number $n, x_{1}(n)=(\operatorname{Bound} 2 \operatorname{Lipschitz}(v \operatorname{veq}(n), X))\left(\$_{1}\right)$ and $x_{1}$ is convergent and $\$_{2}=\lim x_{1}$. For every element $x$ of $X$, there exists an element $y$ of $\mathbb{R}$ such that $\mathcal{P}[x, y]$ by $(23),(33),(26)$, [5, (44)]. Consider $f$ being a function from the carrier of $X$ into $\mathbb{R}$ such that for every element $x$ of $X, \mathcal{P}[x, f(x)]$ from [7, Sch. 3]. Reconsider $t_{1}=f$ as a function from the carrier of $X$ into $\mathbb{R} . t_{1}$ is Lipschitzian by [13, (14)], [11, (12)], (23), (26). For every real number $e$ such that $e>0$ there exists a natural number $k$ such that for every natural number $n$ such that $n \geqslant k$ for every vector $x$ of $X,\left|(\operatorname{Bound} 2 \operatorname{Lipschitz}(v s e q(n), X))(x)-t_{1}(x)\right| \leqslant e \cdot\|x\|$ by [22, (8)], (23), (33), (26). Reconsider $t_{2}=t_{1}$ as a point of DualSp $X$. For every real number $e$ such that $e>0$ there exists a natural number $k$ such that for every natural number $n$ such that $n \geqslant k$ holds $\left\|v \operatorname{seq}(n)-t_{2}\right\| \leqslant e$ by (23), (33), [13, (45)], (24). For every real number $e$ such that $e>0$ there exists a natural number $m$ such that for every natural number $n$ such that $n \geqslant m$ holds $\left\|v \operatorname{seq}(n)-t_{2}\right\|<e$.

(35) Let us consider a real normed space $X$. Then DualSp $X$ is a real Banach space. The theorem is a consequence of (34).

Let $X$ be a real normed space. One can verify that DualSp $X$ is complete. 


\section{Hahn-Banach Extension Theorem}

Let $V$ be a real normed space.

A subreal normal space of $V$ is a real normed space and is defined by

(Def. 16)

(i) the carrier of it $\subseteq$ the carrier of $V$, and

(ii) $0_{i t}=0_{V}$, and

(iii) the addition of $i t=($ the addition of $V) \uparrow($ the carrier of $i t)$, and

(iv) the external multiplication of $i t=$ (the external multiplication of $V) \uparrow(\mathbb{R} \times($ the carrier of $i t))$, and

(v) the normed of $i t=($ the normed of $V)\lceil($ the carrier of $i t)$.

(36) Let us consider a real normed space $V$, a subreal normal space $X$ of $V$, a Lipschitzian linear functional $f$ in $X$, and a point $F$ of DualSp $X$. Suppose $f=F$. Then there exists a Lipschitzian linear functional $g$ in $V$ and there exists a point $G$ of DualSp $V$ such that $g=G$ and $g \uparrow$ (the carrier of $X)=f$ and $\|G\|=\|F\|$. Proof: Reconsider $X_{0}=X$ as a real linear space. Reconsider $f_{3}=f$ as a linear functional in $X_{0}$. Define $\mathcal{F}$ (element of the carrier of $V)=\|F\| \cdot\left\|\$_{1}\right\|$. Consider $q$ being a function from the carrier of $V$ into $\mathbb{R}$ such that for every element $v$ of the carrier of $V, q(v)=\mathcal{F}(v)$ from [7, Sch. 8]. $q$ is a Banach functional in $V$. For every vector $x$ of $X_{0}$ and for every vector $v$ of $V$ such that $x=v$ holds $f_{3}(x) \leqslant q(v)$ by [19, (4)], (26), [6, (49)]. Consider $g$ being a linear functional in $V$ such that $g \uparrow\left(\right.$ the carrier of $\left.X_{0}\right)=f_{3}$ and for every vector $x$ of $V, g(x) \leqslant q(x)$. For every vector $x$ of $V,|g(x)| \leqslant\|F\| \cdot\|x\|$ by [26, (16)], [20, (2)], [19, (5)]. (The bounded linear functionals norm $V)(g) \leqslant$ (the bounded linear functionals norm $X)(f)$. (The bounded linear functionals norm $X)(f) \leqslant \sup \operatorname{PreNorms}(g)$. (The bounded linear functionals norm $X)(f) \leqslant($ the bounded linear functionals norm $V)(g)$.

(37) Hahn-BANACH EXtension theorem (REAL NORMED SPACES):

Let us consider a real normed space $V$, a subreal normal space $X$ of $V$, a Lipschitzian linear functional $f$ in $X$, and a point $F$ of DualSp $X$. Suppose

(i) $f=F$, and

(ii) for every vector $x$ of $X$ and for every vector $v$ of $V$ such that $x=v$ holds $f(x) \leqslant\|v\|$.

Then there exists a Lipschitzian linear functional $g$ in $V$ and there exists a point $G$ of DualSp $V$ such that $g=G$ and $g \uparrow($ the carrier of $X)=f$ and for every vector $x$ of $V, g(x) \leqslant\|x\|$ and $\|G\|=\|F\|$. Proof: Consider $g$ being a Lipschitzian linear functional in $V, G$ being a point of DualSp $V$ such that $g=G$ and $g \uparrow($ the carrier of $X)=f$ and $\|G\|=\|F\| .\|G\| \leqslant 1$. For every vector $x$ of $V, g(x) \leqslant\|x\|$ by [19, (4)], (26). 


\section{REFERENCES}

[1] Grzegorz Bancerek. Monoids. Formalized Mathematics, 3(2):213-225, 1992.

[2] Grzegorz Bancerek. The fundamental properties of natural numbers. Formalized Mathematics, 1(1):41-46, 1990.

[3] Grzegorz Bancerek. The ordinal numbers Formalized Mathematics, 1(1):91-96, 1990.

[4] Józef Białas. Group and field definitions Formalized Mathematics, 1(3):433-439, 1990.

[5] Czesław Byliński. The complex numbers Formalized Mathematics, 1(3):507-513, 1990.

[6] Czesław Byliński. Functions and their basic properties Formalized Mathematics, 1(1): 55-65, 1990.

[7] Czesław Byliński. Functions from a set to a set. Formalized Mathematics, 1(1):153-164, 1990.

[8] Czesław Byliński. Partial functions Formalized Mathematics, 1(2):357-367, 1990.

[9] Czesław Byliński. Some basic properties of sets. Formalized Mathematics, 1(1):47-53, 1990.

[10] Noboru Endou, Yasumasa Suzuki, and Yasunari Shidama. Real linear space of real sequences. Formalized Mathematics, 11(3):249-253, 2003.

[11] Jarosław Kotowicz. Real sequences and basic operations on them Formalized Mathematics, 1(2):269-272, 1990.

[12] Jarosław Kotowicz. Convergent sequences and the limit of sequences. Formalized Mathematics, 1(2):273-275, 1990 .

[13] Jarosław Kotowicz. Convergent real sequences. Upper and lower bound of sets of real numbers. Formalized Mathematıcs, 1(3):477-481, 1990.

[14] Eugeniusz Kusak, Wojciech Leończuk, and Michał Muzalewski. Abelian groups, fields and vector spaces Formalized Mathematics, 1(2):335-342, 1990.

[15] Anna Justyna Milewska. The Hahn Banach theorem in the vector space over the field of complex numbers Formalızed Mathematıcs, 9(2):363-371, 2001.

[16] Adam Naumowicz. Conjugate sequences, bounded complex sequences and convergent complex sequences Formalızed Mathematıcs, 6(2):265-268, 1997.

[17] Bogdan Nowak and Andrzej Trybulec. Hahn-Banach theorem. Formalized Mathematics, 4(1):29-34, 1993.

[18] Henryk Oryszczyszyn and Krzysztof Prażmowski. Real functions spaces. Formalized Mathematics, 1(3):555-561, 1990.

[19] Jan Popiołek. Some properties of functions modul and signum. Formalized Mathematics, 1(2):263-264, 1990.

[20] Jan Popiołek. Real normed space, Formalized Mathematics, 2(1):111-115, 1991.

[21] Yasunari Shidama. Banach space of bounded linear operators. Formalized Mathematics, 12(1):39-48, 2004.

[22] Yasumasa Suzuki, Noboru Endou, and Yasunari Shidama. Banach space of absolute summable real sequences. Formalized Mathematics, 11(4):377-380, 2003.

[23] Andrzej Trybulec. Binary operations applied to functions. Formalized Mathematics, 1 (2):329-334, 1990.

[24] Andrzej Trybulec. On the sets inhabited by numbers Formalized Mathematics, 11(4): 341-347, 2003.

[25] Wojciech A. Trybulec. Subspaces and cosets of subspaces in real linear space, Formalized Mathematics, 1(2):297-301, 1990.

[26] Wojciech A. Trybulec. Vectors in real linear space Formalized Mathematics, 1(2):291-296, 1990.

[27] Zinaida Trybulec. Properties of subsets. Formalized Mathematics, 1(1):67-71, 1990.

[28] Edmund Woronowicz. Relations and their basic properties Formalized Mathematics, 1 (1):73-83, 1990.

Received March 31, 2014 